\title{
Carpal tunnel syndrome
}

National Diabetes Information Clearinghouse (NDIC)

\section{Definitions}

Neuropathy

Defined by National Diabetes Information Clearinghouse (NDIC)

Diabetes

Defined by National Diabetes Information Clearinghouse (NDIC)

\section{Source}

National Diabetes Information Clearinghouse (U.S.). (2009). The diabetes dictionary. [Bethesda, Md.]: U.S. Dept. of Health and Human Services, National Institutes of Health, National Institute of Diabetes and Digestive and Kidney Diseases, National Diabetes Information Clearinghouse.

A common form of neuropathy occurring with diabetes. Symptoms of carpal tunnel syndrome include nighttime hand tingling and pain and numbness and weakness in the hand and wrist that sometimes radiates up the arm. Carpal tunnel syndrome often affects both wrists and occurs more often in women than men. 